ISSN: 2600-5859

\title{
Planificación territorial, una valoración desde las perspectivas del desarrollo rural endógeno en el caso de la parroquia Malacatos, del cantón Loja.
}

Territorial planning, a valuation from the prospects of endogenous rural development in the case of the Malacatos parish, of the canton Loja.

\author{
Zulay Natalia Jiménez Aguilera ${ }^{1}$
}

Recibido: 17-12-2020 / Revisado: 06-01-2021 /Aceptado: 16-01-2019/ Publicado: 05-02-2021

\begin{abstract}
.
DOI: https://doi.org/10.33262/concienciadigital.v4i1.1.1549

Introduction. Endogenous development emerges as an alternative to traditional development approaches, to incorporate above all a participatory and territorial vision, which goes beyond purely agrarian perspectives and introduces social, environmental, political, economic, institutional connotations, etc. It promotes the search for "progress" based on the assessments and interests of the key actors in the territory and making use of the material and immaterial resources of each locality. Objective. To determine the use of endogenous resources for rural development, which were considered in the territorial planning of the Malacatos parish in the period 2014 - 2019. Methodology. The research design was qualitative, with a descriptive-analytical scope, developed under a case study in the rural Malacatos parish. Semi-structured interviews were applied to the presidents of the local government of the parish, both from the study period and from the current administration; and, to key territorial actors; Likewise, a documentary review of their study period planning instrument was carried out, based on a standardized assessment matrix. Results. The main results show that the parish has the cultivation of sugar cane and tourist activity, as determining endogenous resources and potentialities, however, the territorial planning of the period 2014 - 2019 did not manage to fully focus its interventions on the use of these key elements. Aspects such as the legal competencies of the local government, the low level of associativity of the population, the dispersion of the participatory process and the intervention

\footnotetext{
${ }^{1}$ Universidad Católica de Cuenca, Posgrado, Maestría en Desarrollo Local con mención en Planificación, Desarrollo y Ordenamiento Territorial, Cuenca, Ecuador, zulay.jimenez@est.ucacue.edu.ec, Afiliación https://orcid.org/0000-0001-7866-8746
} 
priorities due to the lack of definition of a comprehensive development model, were some of the variables that contributed in this regard. Conclusion. The territorial planning 2014 - 2019 of Malacatos has some important characteristics to be a generator of endogenous development, however, it has not been able to fully take advantage of the territory's own / identity resources for this purpose.

Keywords: Endogenous rural development, endogenous resources, territorial planning, local government

\section{Resumen.}

Introducción. El desarrollo endógeno surge como una alternativa a los enfoques tradicionales del desarrollo, para incorporarle sobre todo una visión participativa y territorial, que supere perspectivas puramente agrarias e introduzca connotaciones sociales, ambientales, políticas, económicas, institucionales, etc. Promueve la búsqueda del "progreso" a partir de las valoraciones e intereses de los actores clave del territorio y haciendo uso de los recursos materiales e inmateriales propios de cada localidad. Objetivo. Determinar el aprovechamiento de los recursos endógenos para el desarrollo rural, que fueron considerados en la planificación territorial de la parroquia Malacatos en el periodo 2014 2019. Metodología. El diseño de investigación fue cualitativo, con alcance descriptivo analítico, desarrollada bajo un estudio de caso en la parroquia rural Malacatos. Se aplicaron entrevistas semiestructuradas a los presidentes del gobierno local de la parroquia, tanto del periodo de estudio, como de la actual administración; y, a actores territoriales clave; así mismo se realizó la revisión documental de su instrumento de planificación del periodo de estudio, a partir de una matriz de valoración estandarizada. Resultados. Los principales resultados muestran que la parroquia cuenta con el cultivo de la caña de azúcar y la actividad turística, como recursos y potencialidades endógenas determinantes, sin embargo, la planificación territorial del periodo 2014 - 2019 no logró enfocar totalmente sus intervenciones en el aprovechamiento de estos elementos clave. Aspectos como las competencias legales del gobierno local, el bajo nivel de asociatividad de la población, la dispersión del proceso participativo y las prioridades de intervención a causa de la indefinición de un modelo de desarrollo integral, fueron algunas de las variables que aportaron en este sentido. Conclusión. La planificación territorial 2014 - 2019 de Malacatos tiene algunas características importantes para ser generadora de desarrollo endógeno, sin embargo, no ha logrado aprovechar totalmente los recursos propios/ identitarios del territorio para este fin.

Palabras claves: Desarrollo rural endógeno, recursos endógenos, planificación territorial, gobierno local. 


\section{Introducción.}

El desarrollo endógeno surge en medio del debate respecto de las consideraciones puramente económicas o con enfoque ambiental sobre el desarrollo, como una alternativa para repensar las características y condiciones necesarias para lograr "progreso" en un determinado espacio. Según Vásquez (2007) tuvo sus inicios en Europa, en la década de los 80.

Las reflexiones que llevaron a repensar la idea de desarrollo involucraron cuestiones ambientales, sociales, culturales y políticas, principalmente. En ese contexto surgieron nuevas conceptualizaciones del desarrollo, como el enfoque sostenible, por ejemplo, que básicamente discutía la necesidad de mirar al desarrollo desde la perspectiva medio ambiental, para garantizar la disponibilidad de los recursos naturales del planeta, para las actuales y futuras generaciones.

Así mismo surgen otras reflexiones sobre territorialidad, que resultan bastante pertinentes al hablar de desarrollo local y rural. Según Massiris (2015) coincidente con Massiris (2012) al incorporar el concepto de territorio a las definiciones de desarrollo es posible ampliarlo y verlo desde una perspectiva integral y multidimensional, porque se analiza la vida de la población desde los ámbitos social, político, ambiental e institucional, valorando sus intereses y libertades.

La corriente del desarrollo endógeno por su parte integra varias de esas reflexiones y ha sido conceptualizado desde diferentes enfoques teóricos (Gómez et al., 2014; Torres et al., 2011), sin embargo, de acuerdo con Quispe (2016) sobre todo trata de ahondar en el rescate de las valoraciones de los actores sociales en la definición y construcción de su propio "desarrollo". Definición que también subyace en el análisis de Briceño, 2018.

Según Moisá y Hernández (2010) el desarrollo rural desde el enfoque de la endogeneidad es visto como aquel proceso que utiliza los recursos humanos y materiales propios de los individuos y el territorio, para pensar/ diseñar su modelo y estrategias de mejoramiento de calidad de vida. Este enfoque "desde abajo" busca superar el modelo tradicional de desarrollo rural en el que la visión agrícola primaba sobre las demás dimensiones que abarcan la ruralidad, bajo la concepción de que su único rol es la provisión de alimentos hacia lo urbano.

En esa línea Moisá y Hernández (2010) sostienen que la discusión del desarrollo rural debería partir de "los procesos emergentes de las comunidades y orientar la planificación a suplir las necesidades de estas..." (p. 516), pues son estos instrumentos los que debe dar cuenta de la realidad de la "ruralidad" más allá de lo agrario. A partir del análisis de 52 veredas de Medellín, el autor asegura que, el considerar a este espacio como un constructo cultural y territorial, puede ser el eje fundamental para que la planificación e intervención pública permitan procesos endógenos con fuertes propiedades emergentes; y, concluye con la importancia de abordar una planificación participativa, real y cercana con las necesidades, 
ISSN: 2600-5859

problemáticas, potencialidades y recursos de las localidades, desde una visión multidimensional y holística.

Así mismo, Valenciano y Carretero (2006) inspirado en el modelo europeo, después de debatir sobre las consideraciones del desarrollo rural, bajo los enfoques de endogeneidad, integralidad o localía, considera que éste "resulta del esfuerzo de concertación y planificación emprendido por el conjunto de los actores locales..." (p. 60).

Sin duda, los instrumentos de planificación territorial son herramientas válidas para abordar esta visión de desarrollo, en la medida en que permiten clarificar lo que se pretende alcanzar en un futuro cercano o lejano y los medios/ estrategias y recursos necesarios para lograrlo. Según Gómez et al. (2018) ésta sustenta y prevé una estructura para la toma de decisiones.

Según Massiris (2020), en América Latina las ideas sobre planificación y ordenamiento territorial tienen sus inicios en los años 30, con los primeros esfuerzos en planificación física espacial especialmente de corte urbanística, cuya cronología empieza con México en 1933 a través del Plan Regulador de la Ciudad de México y termina en Brasil, en 1979 con la Ley 6766 regulatoria del uso del suelo urbano en cabeza de municipio.

Así mismo, según la misma fuente, en las próximas décadas ya se registran avances en la incorporación de enfoques ambientales (cuencas hidrográficas), en sintonía con las reflexiones que sobre desarrollo sostenible se impulsaban en la región, así como sobre el abordaje del ordenamiento en territorio marino costeros y de riesgos.

Finalmente, con la llegada del nuevo siglo se introducen algunas concepciones pensadas sobre todo en el ordenamiento a nivel rural y el enfoque institucional para abordarlo. De manera específica en Ecuador, con la expedición de una nueva Constitución en 2008, el Código Orgánico de Planificación y Finanzas Públicas (COPYFP) y el Código Orgánico de Planificación y Desarrollo (COOTAD) en 2010, se establece un marco normativo amplio en temas de planificación y ordenamiento territorial a nivel local, haciendo obligatoria su construcción y ejecución para todos los Gobiernos Autónomos Descentralizados (GAD).

Estos cuerpos normativos, más allá de establecer la planificación territorial como una obligatoriedad competencial de los gobiernos locales en todos los niveles, instaura un modelo de descentralización en el país -como un proceso obligatorio, progresivo y definitivo- que buscaba otorgar mayor poder a los territorios y afianzar sus capacidades para gestar su desarrollo. En función de aquello se creó un Sistema Nacional Descentralizado de Planificación Participativa y un Consejo Nacional de Competencias, cuyo rol estaba precisamente en organizar el proceso descentralizador y -entre otras cosas- transferir y regular competencias a los GAD.

Es así como, a partir de entonces, en el marco de su autonomía, los GAD empezaron a desarrollar Planes de Desarrollo y Ordenamiento Territorial (PDOT) -especialmente en el 
nivel parroquial rural, pues muchos municipios y gobiernos provinciales ya lo hacían con anterioridad a la expedición de las referidas normas legales-.

Si se parte de la premisa de que, en Ecuador -según la normativa vigente- la planificación territorial es concebida como un medio para alcanzar el desarrollo, es preciso valorar cuan cerca se está hoy en día de consolidar dicha aseveración. Según el art. 41 del COPYFP los PDOT son los instrumentos de planificación que contienen las directrices principales de los GAD respecto de las decisiones estratégicas de desarrollo y que permiten la gestión concertada y articulada del territorio.

Hoy en día ya se registra el tercer ejercicio de planificación territorial, a partir de la normativa vigente, y los retos aún son grandes. Conviene preguntarse si realmente estos instrumentos de planificación están enfocados en el aprovechamiento de los recursos y potencialidades locales, están siendo construidos e implementados desde la visión ciudadana, están considerando un enfoque "desde abajo", tienen una visión multisectorial que supere lo meramente agrario, están logrando articular intervenciones desde diferentes frentes para lograr impactos positivos en la calidad de vida de los habitantes; o por el contrario se han convertido en documentos construidos desde el tecnicismo, que poco o nada contribuyen a los objetivos para los que fueron normados.

La Secretaría Técnica Planifica Ecuador (STPE) no ha realizado un estudio o evaluación que permita determinar aquello; y, determinarlo implica una revisión coherente de las intervenciones que se realizan en el territorio en todas sus fases, sin embargo, en un primer momento, es vital centrar la atención en el proceso constructivo que se ha venido desarrollando y los documentos que se han obtenido producto de éste.

Es preciso valorar ¿cuán efectivos han sido estos esfuerzos de planificación territorial en la generación de desarrollo local? ¿las estrategias son realmente pertinentes con las cadenas de valor territorial? ¿se ha generado vinculación y aprendizaje social? ¿se ha abordado el territorio desde una mirada multidimensional? ¿se han focalizado las vocaciones de los recursos territoriales? ¿se han generado redes/ sinergias entre actores territoriales?, ¿ha existido apropiación de los beneficios del desarrollo por parte de la población?, etc.

Por lo expuesto, se plantea la presente propuesta de investigación que aterrice toda esa problemática, en un estudio de caso que permita determinar el aprovechamiento de los recursos endógenos para el desarrollo rural, que han sido considerados en la planificación territorial.

Para ello, se parte de la identificación de las tendencias teóricas y empíricas sobre la planificación territorial del desarrollo rural endógeno y las características que debe tener ésta para generarlo; a fin de posteriormente caracterizar el aprovechamiento de los recursos y potencialidades endógenas de Malacatos para generar desarrollo rural; y, finalmente 
proponer líneas de acción que permitan mejorar estos ejercicios de planificación, desde la visión de la endogeneidad.

\section{Metodología}

El presente estudio es de naturaleza cualitativa, con alcance descriptivo - analítico. Es cualitativa porque analiza en profundidad el estudio de caso de la parroquia rural de Malacatos, respecto de los recursos endógenos característicos que posee y su aprovechamiento en la planificación territorial.

$\mathrm{Su}$ alcance es descriptivo - analítico en la medida en que logra identificar los recursos endógenos que potencian el desarrollo rural de la parroquia, caracterizar sus usos, limitaciones y ventajas singulares, para posteriormente examinar y valorar en profundidad su aprovechamiento en la planificación territorial que impulsó el GAD parroquial.

Al tener este alcance investigativo se hizo uso de técnicas como la entrevista semiestructurada y la revisión documental. Para el primer caso se diseñaron tres cuestionarios diferentes enfocados en recabar información para el cumplimiento de los objetivos previstos y dirigidos a públicos diferentes, de acuerdo con siguiente detalle:

- Presidente del GAD parroquial de Malacatos periodo 2014 - 2019, para conocer de primera mano la valoración del ejercicio de planificación territorial del periodo 2014 -2019, considerando sus fases de construcción, ejecución, seguimiento y evaluación, desde las perspectivas de generación de desarrollo endógeno; así como los recursos y potencialidades que se priorizaron para el destino de los recursos públicos.

- Presidente del GAD parroquial de Malacatos periodo 2019 - 2023, para conocer desde la perspectiva de la actual autoridad las características técnicas que deben tener los instrumentos de planificación parroquial para generar desarrollo rural en sus diferentes dimensiones, así como las perspectivas, recursos y potencialidades de desarrollo endógeno que se visualizan para la parroquia.

- Actores sociales clave de la parroquia, para identificar los recursos endógenos más representativos que tiene el territorio, caracterizarlos (uso, limitaciones, competitividad y sostenibilidad) y valorar su nivel de aprovechamiento para la consecución de los objetivos de desarrollo. La selección obedeció al criterio de pertenencia a organizaciones sociales, directivas barriales y consejo de planificación local.

La técnica de la revisión documental fue aplicada al PDOT de Malacatos, periodo 2014 2019 utilizando una matriz estructurada, construida a partir de los estándares planteados por Sáenz (2006), y ajustada en función del modelo de Cazanelli (2004).

El modelo de Sáenz (2006) tiene varias aristas, pues abarca el desarrollo local en sentido amplio, sin embargo, para efectos de esta investigación, se utilizó el ámbito de la 
planificación, en dónde el autor considera que se mide la existencia de: un documento del plan elaborado, diagnóstico, visión, líneas estratégicas, proyectos y presupuesto para su cumplimiento.

Además, se toman algunos elementos de los estándares que el autor propone para participación y construcción de tejido social, que son aplicables a los procesos de planificación territorial.

Los estándares se organizan en la siguiente escala secuencial planteada por Sáenz (2006):

No hay resultado alguno respecto al indicador: Se usa el "0" (cero), como una alternativa de respuesta cuando hay ausencia de logro.

Resultado bajo respecto al estándar esperado (1): Esto puede significar que todavía este elemento está en una fase inicial o de implementación o no ha logrado todavía alcanzar un estándar aceptable.

Resultado medio (2): Significa que el elemento medido ha alcanzado un nivel que puede ser considerado "en proceso" o aceptable, aunque no haya llegado a niveles de excelencia. En general este estándar muestra que la condición de desarrollo medida se está desenvolviendo.

Estándar alto (3): Quiere decir que este elemento ha logrado un funcionamiento o resultado de calidad y, por lo tanto, coadyuva al logro de un proceso de desarrollo sostenible e institucionalizado. (p. 6)

\section{Resultados.}

Malacatos es una de las trece parroquias rurales del cantón Loja, se encuentra ubicada a 33 $\mathrm{km}$ de la cabecera cantonal y provincial y tiene una población proyectada de 9076 habitantes, al 2020 .

Según Instituto Nacional de Estadísticas y Censos (INEC, 2010), tiene un índice de pobreza por Necesidades Básicas Insatisfechas (NBI) de 77,75\%, muy superior al del cantón Loja, que se ubica en 43,59\% para el mismo año de referencia; pero, muy por debajo de los índices presentados por la mayoría de las parroquias rurales de la provincia.

$\mathrm{Su}$ población económicamente activa está principalmente dedicada a las actividades de agricultura y ganadería $(41,41 \%)$, seguida de la construcción $(10,40 \%)$ y comercio $(9,76 \%)$. A nivel de indicadores educacionales, registra una tasa de analfabetismo de 5,70\%, 8,25 años de escolaridad promedio de la población de 24 años y más y tasas de asistencia a clases que van desde el $96 \%$ en el nivel primario, hasta el 17,44 a nivel de educación superior. 
Recursos endógenos de Malacatos: caracterización y potencial de aprovechamiento

Bajo las reflexiones teóricas que conciben al desarrollo endógeno como un proceso "desde adentro/ desde abajo", Gómez et al. (2018) lo conceptualizan como un "modelo de desarrollo interno, que toma en cuenta la competencia comparativa de cada región o país" bajo "la integración de todos sus recursos" y la entrega del poder a las comunidades organizadas.

En la misma línea Moisá y Hernández (2010) añaden otras visualizaciones, desde el enfoque de libertades -trabajado por Amartya Sen- resaltando que este proceso de desarrollo territorial rural no puede venir de modelos "exitosos" impuestos desde las localidades que se dicen ser "desarrollados", sino más bien desde la cosmovisión, recursos y características de los mismos habitantes. En ese sentido el "desarrollo" como concepto va a estar definido en función de los intereses y libertades que pretenda alcanzar ese territorio en particular.

En ese sentido, tomando en cuenta el criterio de los actores sociales y autoridades locales entrevistadas, se puede determinar que los recursos endógenos que tienen la capacidad de generar desarrollo en Malacatos a partir de su aprovechamiento, están relacionados con el cultivo de caña de azúcar, tanto para producción de panela como aguardiente; el cultivo de café, la elaboración de ladrillos, la actividad turística y la explotación de pétreos, en menor medida. De éstos, los principalmente priorizados son: el cultivo de la caña de azúcar y el turismo.

De acuerdo con GAD Malacatos (2018), en la parroquia 2433,58 hectáreas están dedicadas a la actividad agrícola, en donde el cultivo de caña de azúcar es el más amplio (728,03 ha). Ello está relacionado con las características propias de un territorio rural como la parroquia objeto de estudio, que -de acuerdo con INEC (2010)- presenta el 41,51\% de su población dedicada a actividades de agricultura, ganadería, silvicultura y pesca; y, donde se registra un importante flujo de relaciones urbano - rurales, por su cercanía con la cabecera cantonal y provincial; lo que a su vez influye en el potencial turístico que se puede generar, en torno a sus atractivos naturales, gastronómicos y religiosos.

En ese contexto, y considerando los mecanismos utilizados por Canzanelli (2004) en su estudio sobre la valorización del potencial endógeno, competitividad territorial y lucha contra la pobreza, se puede determinar la siguiente caracterización del aprovechamiento de los recursos endógenos de Malacatos, para generar desarrollo rural:

Según el modelo en mención, por cada uno de los recursos endógenos identificados es necesario analizar su aprovechabilidad ${ }^{2}$, competitividad $^{3}$ y sostenibilidad ${ }^{4}$.

\footnotetext{
${ }^{2}$ Se toma en cuenta la existencia o no de limitantes para su aprovechamiento y el uso más apropiado para potenciar su aprovechamiento.

${ }^{3}$ Se considera las características diferenciales del recurso que le otorgan ventajas potenciales propias o territoriales.

${ }^{4}$ Se valora la capacidad del recurso para mantener en el tiempo su aprovechamiento.
} 
Cultivo de caña de azúcar: es un producto que al momento está siendo cultivado tanto para la elaboración de aguardiente, como para panela. En la mayoría de los casos los agricultores que se dedican a esta actividad comercializan la materia prima hacia los propietarios de fábricas para la elaboración de aguardiente artesanal o panela; sin embargo, en la actualidad ya se cuenta con pequeños emprendimientos que buscan diversificar los productos derivados mediante la agregación de valor. Se cuenta con la primera embotelladora de anisado (aguardiente preparado con carnes y anís); y, con la elaboración de panela granulada.

De acuerdo con el levantamiento de información con actores locales, el uso más apropiado para el aprovechamiento de este recurso es la agregación de valor y diversificación de derivados de la panela, con miras a expandir mercados nacionales e internacionales. Para ello, se requiere mejorar la productividad de los cultivos a través del análisis de suelo para la incorporación de los nutrientes que el producto necesita, la mejora de las semillas de acuerdo con las condiciones físico - naturales del territorio, la tecnificación de los procesos; y, el fomento del nivel organizativo de los productores.

Hoy en día, el potencial aprovechamiento de este recurso para la generación de desarrollo se ha visto limitado por varios aspectos. Al ser la venta de la materia prima la principal línea de generación de ingresos dentro del cultivo de este producto está sujeta a las variaciones estacionales de precios, que a su vez imposibilitan cubrir con los gastos operativos derivados del proceso productivo (compra de insumos, pago de mano de obra) y recuperar inversiones. Ello adicionalmente, influye negativamente en el interés de los productores para asociarse, implementar emprendimientos y sobre todo mejorar el equipamiento e insumos necesarios para cultivar la caña de azúcar y producir sus derivados.

Por otro lado, la inestabilidad en los precios también está relacionada con la intermediación en los procesos de comercialización de los productos y la necesidad de ampliar los mercados de destino.

Según la misma fuente, este producto es potencialmente competitivo por los volúmenes de producción, derivados de las extensiones de terreno dedicadas a su cultivo; la aptitud físico natural del territorio para su cultivo, la calidad de la panela que se produce, por su alto nivel de dureza (que a su vez asegura mayores periodos de conservación), el aroma y sabor. Así mismo Malacatos goza de un reconocimiento importante a nivel local y nacional, como territorio productor de aguardiente y panela, por excelencia.

Por último, la sostenibilidad que se le atribuye es de orden ambiental y económica. En el primer caso, las características propias del cultivo hacen que no se requiera el uso de gran cantidad de químicos o fungicidas, en comparación con otro tipo de cultivos; así mismo en la parroquia se desarrolla un proceso de aprovechamiento limpio, pues no se quema la plantación una vez que se está listo para su corte (a diferencia de los mecanismos utilizados en territorios cercanos). 
En el segundo caso, se considera que el recurso es sostenible económicamente siempre y cuando se de valor agregado a los productos y con ello mayor estabilidad en los precios.

Turismo: Malacatos es una parroquia que cuenta con algunos recursos para el aprovechamiento de la actividad turística. A nivel de atractivos naturales se pueden mencionar cascadas y montañas desde donde se pueden apreciar singulares paisajes, senderos y hosterías para el disfrute y recreación. En lo gastronómico se destaca por la gran cantidad de pequeños emprendimientos de venta de carne preparada de cerdo, a lo largo de la vía de ingreso principal de la parroquia, así como otros platos típicos comercializados principalmente en ferias. En el ámbito religioso se caracteriza por ser la única parroquia a nivel local que concentra 31 días de festividades continuas durante el mes de agosto, en honor a la imagen de su patrono. Otro elemento estratégico de este recurso es su capacidad de convertir a Malacatos en un territorio que atrae población -especialmente de la ciudad de Loja-, por ser considerado un lugar de descanso cercano o de segunda residencia.

Para potenciar su aprovechamiento, los actores sociales consideran vital impulsar el turismo religioso, gastronómico, vacacional y de atractivos naturales. Para ello es preciso la ampliación de oferta de destinos turísticos, la infraestructura pública y privada que permita su disfrute, los servicios complementarios (alimentación, alojamiento, etc.,) y la conectividad vial que facilite el acceso, a través de una fuerte articulación entre el sector público y privado.

Otra línea de trabajo que potencia su aprovechamiento es la promoción y difusión del recurso con enfoque comunitario rural, que incluya a la población local como principal vocera y conocedora de los atractivos turísticos. Pese a lo anterior, su impulso se ha visto limitado especialmente por la baja inversión de recursos públicos en mejora de infraestructura y servicios que desde el GAD municipal -principalmente- se debería emprender, considerando la asignación competencial; así como las restricciones en términos de movilidad y encuentro social que ha generado la pandemia por el Covid - 19. A nivel de turismo vacacional, se han presentado otras limitantes asociadas al cambio de uso de suelo y fraccionamiento para construcción de fincas vacacionales.

Las ventajas diferenciales que se han identificado en torno a este recurso, están relacionadas con el clima cálido del que goza la parroquia, que la convierten en el lugar ideal para personas de edad avanzada que tienen afectaciones de salud relacionadas con las bajas temperaturas de la ciudad; la cercanía con capital provincial y cantonal; y, visión autóctona e identidad cultural por población nativa, que aún se conserva, a diferencia de territorios cercanos que han sido fuertemente influenciados por culturas extranjeras.

Naturalmente esta actividad es considerada sostenible desde el punto de vista ambiental, por su generación casi nula de contaminación; así como desde el punto de vista económico, por su capacidad para generar dinamización en los comercios y servicios locales. 
ISSN: 2600-5859

A partir de la caracterización anterior, se puede decir entonces que Malacatos dispone de importantes recursos endógenos que potencian su desarrollo, siempre y cuando se generen líneas de acción que permitan su aprovechamiento sostenible y duradero.

\section{Planificación territorial de Malacatos: caracterización y valoración del aprovechamiento de los recursos endógenos}

Al tomar como base las reflexiones teóricas del desarrollo endógeno y las herramientas metodológicas planteadas por Sáenz (2006) para analizar la gestión del desarrollo local a partir de la capacidad de conducir los procesos, más que en los resultados en sí (especialmente en los casos en donde no existen sistemas o indicadores cuantitativos que ayuden a valorarlos) se puede determinar que la planificación territorial de la parroquia Malacatos en el periodo 2014 - 2019 cuenta con algunos elementos importantes que pueden hacerla generadora de desarrollo endógeno, pero también con limitaciones de orden metodológico y práctico que es preciso superar. De acuerdo con la revisión del PDOT de Malacatos del periodo 2014 - 2019 y la entrevista realizada a la presidente del gobierno local, del periodo 2014 - 2019, se tiene los siguientes resultados en la Matriz resultados de la valoración de la planificación territorial en la generación de desarrollo endógeno.

Tabla 1 Matriz resultados de la valoración de la planificación territorial en la generación de desarrollo endógeno.

\begin{tabular}{|c|c|c|c|c|}
\hline Tema & Indicador & $\begin{array}{l}\text { Puntaje } \\
\text { máximo }\end{array}$ & \multicolumn{2}{|c|}{ Valoración } \\
\hline Participación local & ¿El PDOT fue construido de forma participativa? & 3 & 2 & $66,67 \%$ \\
\hline \multirow[t]{2}{*}{ Diagnóstico territorial } & $\begin{array}{l}\text { Existencia de un diagnóstico ambiental, social, cultural, } \\
\text { económico - productivo e institucional. }\end{array}$ & \multirow[t]{2}{*}{6} & \multirow[t]{2}{*}{4} & \multirow[t]{2}{*}{$66,67 \%$} \\
\hline & Grado de uso y conocimiento de sistemas de información. & & & \\
\hline \multirow{3}{*}{ Enfoque de resultados } & Existencia de una visión estratégica de desarrollo parroquial & \multirow{3}{*}{9} & \multirow{3}{*}{4} & \multirow{3}{*}{$44,44 \%$} \\
\hline & $\begin{array}{l}\text { ¿El PDOT se enfoca en los recursos endógenos de la } \\
\text { parroquia? }\end{array}$ & & & \\
\hline & $\begin{array}{l}\text { Existencia de líneas base, metas e indicadores en la propuesta } \\
\text { del PDOT. }\end{array}$ & & & \\
\hline Relación multinivel & $\begin{array}{l}\text { ¿El PDOT prevé articulación con los diferentes niveles de } \\
\text { gobierno? }\end{array}$ & 3 & 3 & $100 \%$ \\
\hline \multirow[t]{2}{*}{ Aprovechabilidad } & $\begin{array}{l}\text { Porcentaje de proyectos del PDOT relacionados con los } \\
\text { recursos endógenos de la parroquia. }\end{array}$ & \multirow[t]{2}{*}{6} & \multirow[t]{2}{*}{2} & \multirow[t]{2}{*}{$33,33 \%$} \\
\hline & $\begin{array}{l}\text { El PDOT identifica condiciones para el aprovechamiento de } \\
\text { recursos endógenos de la parroquia. }\end{array}$ & & & \\
\hline
\end{tabular}

Nota. Datos calculados con base en los resultados de la revisión del PDOT de Malacatos 2014 - 2019 y entrevistas a presidentes de GAD. Elaboración propia. 
Figura 1 Valoración de la planificación territorial en la generación de desarrollo endógeno.

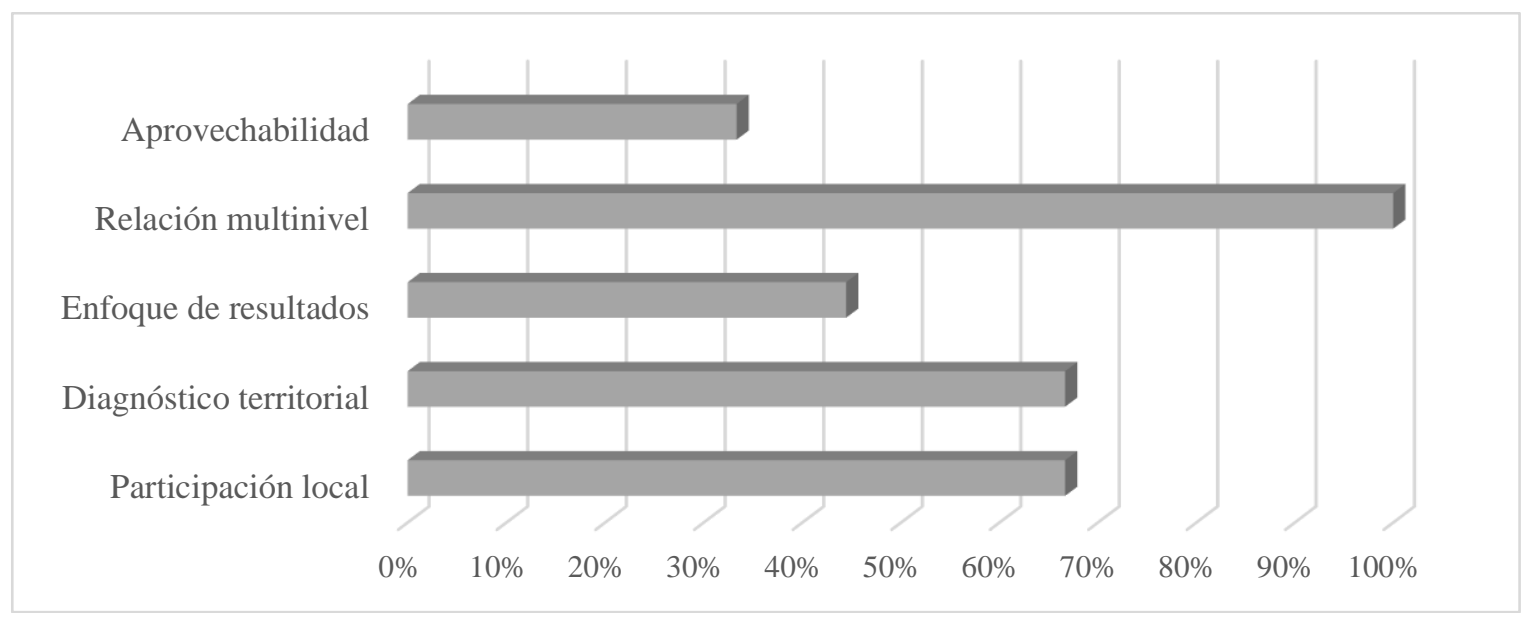

Nota. La figura muestra los resultados de la aplicación de los estándares contenidos en la matriz de revisión del PDOT 2014 - 2019. Fuente: Elaboración propia.

\section{Participación local}

En el desarrollo endógeno la participación de los actores locales es una característica predominante, pues se trata precisamente de generar procesos de bienestar, progreso y calidad de vida a partir de los intereses, valoraciones y expectativas de los habitantes.

Desde la perspectiva de la Innovación rural como teoría en construcción -y en el marco de la contribución del desarrollo endógeno a ésta- Burgos y Bocco (2020), coincidente con Quispe y Ayaviri (2013), Sáenz (2006), sostienen que "el enfoque endógeno destaca el papel de los actores locales, a quienes se asigna un rol protagónico en la integración de redes que propicien la emergencia de lo nuevo, en el aprendizaje, y en la regulación para una apropiación sustentable de los recursos territoriales" (p. 232).

En ese sentido, Sáenz (2006), dentro de los estándares para la medición de la gestión del desarrollo local, plantea que "la primera condición de desarrollo es la participación de actores locales" (p. 7). En lo aplicable a la planificación territorial -y considerando las disposiciones del COPYFP del Ecuador, art. 28 y 29- , es preciso valorar la participación de la ciudadanía en las diferentes fases del PDOT.

Al respecto la planificación de Malacatos, periodo 2014 - 2019 tiene una valoración de 2, pues para el diseño del PDOT se realizaron mesas de trabajo con ciudadanía, organizando los 52 barrios, en 8 sectores o áreas territoriales, a fin de reducir la dispersión y nutrir los aportes. 
Según S. Rodríguez (comunicación personal, 10 de diciembre, 2020) los espacios participativos se realizaron para la fase de diagnóstico y propuesta, a través de la identificación de problemas/ potencialidades y priorización de intervenciones. En la primera parte, la información fue útil para caracterizar al territorio y analizar las necesidades reconocidas por sus habitantes; mientras que, en la segunda parte, la actividad estuvo encaminada a identificar las problemáticas o potencialidades más urgentes -según el criterio ciudadano-, que necesitaban ser incluidas dentro de la planificación territorial, para su gestión.

Se contó con informe favorable del Consejo de Planificación Local (CPL), de forma previa a la aprobación del PDOT por parte del órgano legislativo, en cumplimiento a la disposición legal contenida en el cuerpo legal referido líneas arriba.

\section{Diagnóstico territorial}

Para que la generación de desarrollo rural endógeno supere la visión meramente agrícola y responda a las conceptualizaciones multi e intersectoriales que proponen autores como Schejtman (2010) o Ballesteros et al. (2013), - estos últimos inspirados en el modelo de Boisier- es preciso que la planificación territorial que lo soporta tenga también estas características.

En ese sentido y tomando en consideración las orientaciones metodológicas que se realizaron por la entonces Secretaría Nacional de Planificación y Desarrollo (SENPLADES, 2015), respecto de formular los PDOT en componentes sectoriales, se puede mencionar que efectivamente, la planificación territorial de Malacatos, del periodo 2014 - 2019 se actualizó bajo un enfoque multi sectorial, incluyendo aspectos: biofísicos, socio culturales, económico productivos, de asentamientos humanos, conectividad, energía y conectividad; y, político institucional; cuya información trata de ser explicada -en más de un componente- desde la perspectiva de los datos duros y alimentada por el criterio ciudadano.

Así mismo, el diagnostico parroquial se sustenta en datos cuantitativos tomados de fuentes oficiales y de cualitativos derivados de los espacios participativos, especialmente el acápite que caracteriza y problematiza el enfoque de género.

Por otro lado, otro de los estándares definidos para el análisis de la planificación territorial, está relacionado con los sistemas de información. En el ámbito del ordenamiento territorial y, según el análisis de Cavuoto (2009) los sistemas de información territorial pueden constituirse en recursos -aunque no indispensables, si- muy deseados para potenciar los procesos de planificación. Su uso es importante no solo para describir, sino sobre todo para medir, proyectar y monitorear a lo largo del tiempo.

$\mathrm{Al}$ respecto se puede mencionar que el GAD de Malacatos no cuenta con un sistema de información local, ni es parte de otro que se administre desde otra entidad, por ello la 
información para la actualización del PDOT 2014 - 2019 fue tomada del Sistema Nacional de Información y ministerios sectoriales como: MSP, MINEDUC, MIES, SRI, SENATEL, CNT, CNE, SEPS, SENAGUA, INEC, IGM, IEE, CLIRSEN, MAGAP, SINAGAP. Además, se tomó información del PDOT del GAD cantonal, realizado en 2015, del Plan Provincial de Riego y Drenaje, construido por el GAD provincial en 2014 y del PDOT parroquial del 2011.

\section{Enfoque de resultados}

Si se parte del precepto conceptual de que la planificación constituye un medio para alcanzar el desarrollo, porque sustenta y prevé una estructura para la toma de decisiones (Gómez et al., 2018), se puede entender entonces que, en el contexto de la endogeneidad, la planificación territorial debe estar relacionada con los recursos endógenos existentes o potenciales.

En ese sentido, se puede mencionar que el PDOT 2014 - 2019 de Malacatos únicamente describe de forma general los recursos endógenos de la parroquia, especialmente en el diagnostico biofísico y económico productivo. En la síntesis de problemas y potencialidades se prioriza la problemática de empleo en el cultivo de caña de azúcar; y la degradación ambiental provocada por la explotación de pétreos; así como la potencialidad del incremento de servicios y comercio asociados al turismo; sin embargo, no centra su atención en la priorización para el desarrollo de intervenciones que puedan potenciarlos. Según S. Rodríguez (comunicación personal, 10 de diciembre, 2020), las causas están asociadas a temas competenciales, limitaciones para su aprovechabilidad y presupuesto.

Por otro lado, el enfoque de resultados también se puede valorar desde la posibilidad de contar con mecanismos de medición que, en el caso de la planificación territorial, estarían dados por la definición de metas e indicadores que evidencien -al cabo del periodo de gestiónresultados palpables.

En el caso del PDOT 2014 - 2019 de Malacatos sus objetivos estratégicos cuentan con líneas base, metas e indicadores, pero éstos no mantienen una estrecha vinculación con las intervenciones planteadas, por lo que, al finalizar la periodicidad de la meta, será complejo su evaluación; así mismo en muchos de los casos no se define línea base, por lo que la meta planteada no tiene un sustento para su proyección.

Además, tampoco se evidencian fuentes de información para medir el avance o cumplimiento de las metas, especialmente en aquellas cuyas intervenciones superan el ámbito de acción del GAD parroquial, por ser competencia del nivel cantonal o provincial.

\section{Relación multinivel}

Al considerar que el desarrollo endógeno tiene de forma implícita la variable territorial, se puede entender que estos procesos no están anclados de forma única a las intervenciones que puede realizar el gobierno local que lo fomenta, sino que la intervención de todos los actores 
es por sobre todo su esencia. En palabras de Sánchez (2015) es importante consolidar "redes colaborativas entre municipios, en las que la participación ciudadana y el aumento de la "cultura territorial" de la población afectada se convierten en imprescindibles" (p. 169).

Estas consideraciones cobran mucha más importancia en territorios parroquiales rurales, cuyos gobiernos locales tiene a su haber muy pocas competencias legales y en el peor de los casos, sus capacidades institucionales no se encuentran fortalecidas.

Es así como, la valoración de la articulación que prevea su instrumento de planificación territorial es crucial a la hora de pensar estratégicamente la generación de desarrollo.

El PDOT 2014 - 2019 de Malacatos identifica actores territoriales y su nivel de articulación con el GAD parroquial. Así mismo en el modelo de gestión se indican los diferentes espacios previstos para la coordinación interinstitucional con el GAD provincial, cantonal y gobierno central, a través de: Consejos sectoriales ciudadanos; mesas de trabajo sectoriales con GAD cantonal; presupuestos participativos con GAD cantonal y provincial; mesa Técnica de articulación META, con el GAD provincial y gestión directa entre autoridades.

En el PDOT se identificaron las problemáticas e intervenciones que servirían de base para la gestión de proyectos ante los demás niveles de gobierno (central, provincial, cantonal); sin embargo, según lo indicado por S. Rodríguez (comunicación personal, 10 de diciembre, 2020), en el periodo 2014 - 2019 se concretaron especialmente las relacionadas con el GAD provincial y central, pues las diferencias políticas trastocaron las intervenciones con el nivel cantonal de gobierno.

Por otro lado, dentro del ordenamiento territorial, el PDOT 2014 - 2019 de Malacatos, asume la categorización y regulación definida por el nivel cantonal, considerando que la competencia de gestión y uso de suelo es exclusivamente de ese nivel.

\section{Aprovechabilidad}

Para valorar el aprovechamiento de los recursos endógenos en el PDOT 2014 - 2019 de Malacatos también se analiza la relación que existe entre el total de proyectos o intervenciones propuestas en el PDOT y la cantidad de éstos que están relacionados con los recursos endógenos de la parroquia.

Al respecto se puede mencionar que existe un total de 51 proyectos $^{5}$, de los cuales únicamente cinco están relacionados con dos de los recursos endógenos de la parroquia: uno relacionado con el control social sobre las actividades mineras (explotación de pétreos); y, cuatro

\footnotetext{
${ }^{5}$ Se analiza el PDOT ajustado, producto de la alineación al nuevo Plan Nacional de Desarrollo 2017 - 2021 , que se aprobó el GAD parroquial en sesión del 6 de abril de 2018, atendiendo las disposiciones de la Resolución Nro. SNPD-065-2017
} 
ISSN: 2600-5859

relacionados con la actividad turística; mientras que ninguna está planteada en función del aprovechamiento del principal cultivo de la parroquia, como es la caña de azúcar.

Así mismo, a excepción de la actividad turística que se la plantea como una potencialidad territorial, en el PDOT se caracteriza a los recursos endógenos y en algunos casos se determinan problemáticas, pero no se generan planteamientos o intervenciones que potencien su aprovechamiento como medios para generar desarrollo, es decir no se evidencia una congruencia clara y directa entre las problemáticas/ potencialidades del diagnóstico y las intervenciones planteadas en la propuesta.

Según S. Rodríguez (comunicación personal, 10 de diciembre, 2020), las limitaciones para la priorización de proyectos enfocados en los recursos endógenos están relacionadas con temas competenciales del nivel parroquial, diferencias políticas con nivel cantonal, recursos económicos y niveles de asociatividad e interés de la población beneficiaria.

Según los actores sociales entrevistados, efectivamente la mayoría coincide en que no se identifican intervenciones relacionadas con los recursos endógenos de la parroquia; y, en general lo ejecutado en el periodo de estudio, se vio afectado por las diferencias políticas entre niveles de GAD, la disminución de recursos públicos y sobre todo por la poca integralidad de las acciones implementadas, pues era preciso que se considere el acompañamiento en todo el ciclo productivo y una visión de desarrollo integral y a largo plazo que las cobije.

\section{Líneas de acción para generar desarrollo endógeno desde la planificación territorial}

- Desde la perspectiva de la integralidad: Si se parte de la premisa de que la base del desarrollo endógeno es la visión de los ciudadanos y demás actores locales que son parte de ese territorio, se puede advertir que la planificación territorial que se geste como un medio para alcanzar este fin, debe necesariamente incorporar esa perspectiva.

En ese sentido y considerando la experiencia analizada para el estudio de caso se plantea la necesidad de incorporar un enfoque participativo en todas las fases que integran la planificación territorial, a partir de un primer ejercicio de identificación de recursos endógenos.

A nivel práctico el enfoque participativo debe ser capaz de superar un diagnóstico sectorial de tipo descriptivo en donde los actores territoriales describan un listado de problemas y necesidades a cubrir, para migrar hacia espacios de análisis de recursos y potencialidades locales, que permitan construir un modelo de desarrollo territorial integral, más que intervenciones aisladas que solucionen necesidades puntuales de un barrio o comunidad.

La planificación territorial debe entenderse como un instrumento de alcance integral, que va más allá de las acciones competenciales que tienen los gobiernos locales. Si 
bien es cierto las experiencias de PDOT en Ecuador han estado ligadas a las disposiciones legales de lo que puede hacer un GAD según la Constitución y el COOTAD, esto no tiene por qué poner en riesgo la integralidad del territorio en análisis y mucho menos la visión de desarrollo a alcanzar.

Para lograrlo es preciso que los PDOT definan líneas de acción integrales, que busquen enfocar los esfuerzos de todos los actores públicos y privados hacia la consecución de un modelo de desarrollo integral que recoja los recursos endógenos de la parroquia. Para ello, a partir de la identificación de los recursos y potencialidades endógenas del territorio, como primer paso del proceso planificador y del involucramiento ciudadano, se debe propender a la conformación de cadenas de valor territorial, es decir entramados coherentes y complementarios de actividades económicas interrelacionadas alrededor de los recursos endógenos potencialmente aprovechables, competitivos y sostenibles. Sobre éstos se define el modelo territorial deseado y las intervenciones de desarrollo necesarias para alcanzarlo.

- Desde la perspectiva de la complementariedad: Si bien es cierto la planificación territorial parroquial -en Ecuador- tiene ciertas diferencias con la planificación del nivel cantonal o provincial, especialmente por el rol que cumple cada gobierno local en la generación de desarrollo y el ordenamiento territorial de las respectivas localidades; ello no tendría que significar que una es más generadora que otra de desarrollo.

Se parte de la premisa que se planifica para el territorio y no para el gobierno local. Por ello, en atención a las disposiciones legales que indican que el uso y gestión de suelo corresponde al nivel cantonal (clasificación y subclasificación) y el modelo productivo al nivel provincial, es preciso que el gobierno parroquial establezca un mecanismo claro de articulación con el gobierno cantonal y provincial al que pertenece, para que se defina y alimente desde su visión subsidiaria, el Modelo Territorial Deseado de su PDOT; se incorpore los intereses y líneas de acción de la parroquia en las regulaciones y PDOT del cantón y provincia; y, sobre todo, se sienten las bases normativas y de inversión para la definición de los proyectos e intervenciones que necesite la parroquia y que se planteen las planificaciones territoriales respectivas.

De esta forma el proceso de planificación territorial se trabajaría sobre la base de la complementariedad entre los diferentes niveles de gobierno que intervienen en un mismo territorio, se evitaría desaciertos de articulación por diferencias políticas entre autoridades; y, se subsanaría las limitaciones en términos de incongruencia entre el diagnóstico sectorial identificado/ caracterizado y la propuesta de intervenciones.

Respecto del último punto es imprescindible lograr una vinculación entre las fases del PDOT, de tal forma que el análisis de diagnóstico se refleje en las propuestas normativas y de inversión que se planteen; y, por ende, en el modelo de gestión que las implemente. Ello se logra al definir en la primera fase los recursos y 
potencialidades endógenas a aprovechar bajo el esquema de cadena de valor territorial, que a su vez definirá las intervenciones de la propuesta.

- Desde la perspectiva de la organización: La normativa ecuatoriana plantea que los PDOT deben actualizarse de forma obligatoria al inicio del periodo de gestión de las autoridades locales, hasta el primer año; sin embargo, no existe disposición expresa que diferencie los periodos de actualización para cada nivel de gobierno, dejando a libertad de los gobiernos locales el inicio y finalización de éste.

Esta discreción temporal genera complicaciones serias en términos de articulación territorial, tanto entre niveles de gobierno (provincial, cantonal, parroquial), como entre territorios circunvecinos, especialmente en la definición de la clasificación y subclasificación del suelo, el modelo territorial deseado y las intervenciones territoriales cuando existen recursos o problemáticas de abordaje mancomunado.

En ese sentido, es preciso lograr coherencia organizativa en el proceso de actualización de los PDOT. Para ello a nivel normativo se debe establecer un orden temporal de inicio y finalización del proceso de actualización de los PDOT cuando se tenga nuevas autoridades, considerando como punto de partida las definiciones cantonales sobre uso y gestión del suelo; momento en el cual se hace determinante la participación del nivel parroquial y provincial para alimentar el proceso de ordenamiento territorial.

El hecho de que no se organice este proceso, complica profundamente la vinculación del ordenamiento territorial (con las definiciones sobre el uso y gestión del suelo) con la definición del modelo productivo; y, por ende, con la implementación de la planificación territorial parroquial.

Por otro lado, con la finalidad de integrar las acciones que se ejecuten sobre un mismo territorio y partiendo de la premisa de que el abordaje del desarrollo territorial es por sobre todas las cosas multidimensional, es necesario que la planificación territorial que fomente el nivel parroquial guarde estrecha sintonía y armonización con las planificaciones sectoriales que impulsa el gobierno central a través de sus diferentes carteras de Estado. Las intervenciones en términos sociales, ambientales, políticos, de seguridad ciudadana, etc., deben guardar correspondencia con las cadenas de valor territorial y modelo de desarrollo deseado que se hayan definido en la planificación territorial, en el contexto de la generación de desarrollo endógeno.

\section{Conclusiones.}

- La planificación territorial puede ser vista como un medio para alcanzar el desarrollo, considerando que la función de ésta es proporcionar un esquema claro y objetivo para la toma de decisiones estratégicas del desarrollo. Al incorporar la visión de endogeneidad al concepto de desarrollo, es preciso también que la planificación 
territorial asuma esos preceptos teórico-conceptuales para ser generadora de desarrollo endógeno.

En ese sentido, a partir de diferentes reflexiones teórico - empíricas revisadas, se considera que para que la planificación territorial sea generadora de desarrollo endógeno, debe: haber integrado la visión, perspectivas e intereses de los actores territoriales como sujetos del desarrollo; abordar el territorio desde un enfoque multisectorial, que supera la visión meramente agraria; sustentar y proyectar sus intervenciones sobre la base de una realidad plenamente medible que muestre continuidad; enfocar el desarrollo a partir de una visión estratégica, medible y propia del territorio, que supera declaraciones genéricas y ambiguas; definir mecanismos de medición del cumplimiento de resultados o cambios en el territorio; fomentar el desarrollo del territorio más allá de las capacidades y competencias institucionales del gobierno local, incorporando mecanismos claros y prácticos de articulación/ coordinación; y, priorizar el enfoque de intervención en los recursos endógenos identificados, sobre la base de un modelo sostenible de aprovechamiento.

- La parroquia Malacatos al ser un territorio rural con importantes vocaciones productivas, prioriza como sus recursos endógenos el cultivo de la caña de azúcar, como su producto estrella; y el turismo como actividad dinamizadora de la economía local. Ambos recursos son potenciales generadores de desarrollo endógeno, por su capacidad competitiva, su sostenibilidad ambiental y económica y sus características para ser plenamente aprovechables.

En el primer caso, su aprovechamiento debe centrarse en la diversificación de sus productos, la tecnificación de los cultivos y la industrialización del proceso productivo, con miras a la exportación de bienes terminados; mientras que, en el segundo caso, el uso más apropiado se encuentra en el fomento de los atractivos naturales, la gastronomía, el reconocimiento como territorio de segunda residencia y la religiosidad.

- Pese a ello, ha existido un limitado aprovechamiento de los recursos endógenos en la planificación territorial del periodo 2014 - 2019. Situaciones como el poco interés ciudadano para trabajar de forma asociada y las competencias legales establecidas para el GAD parroquial, principalmente, han llevado a que la planificación territorial del periodo 2014 - 2019 no enfoque de manera contundente las intervenciones de desarrollo en los recursos endógenos mencionados. Así mismo, otras limitaciones de orden metodológico y práctico relacionadas con la construcción participativa del instrumento, la vinculación directa entre diagnóstico y propuesta de desarrollo, el enfoque y definición de mecanismos de medición que permitan evidenciar resultados de la planificación y el diseño de proyectos puntuales sin la lógica de un modelo de desarrollo integral, han hecho que el PDOT no se visualice como un verdadero medio para alcanzar el desarrollo rural, y mucho menos se enfoque en el aprovechamiento de los recursos endógenos de la parroquia. 
ISSN: 2600-5859

Finalmente, como consecuencia del análisis referido, existe la necesidad de integrar líneas de acción que permitan fortalecer los esfuerzos de la planificación territorial de Malacatos en la generación de desarrollo endógeno. Para ello es preciso ajustar sus mecanismos participativos, especialmente tratando de evitar la divagación de los aportes sobre un listado de problemáticas puntuales, para migrar hacia reflexiones pensadas a partir de las potencialidades endógenas del territorio y sobre la base del análisis de un modelo de desarrollo que apunte a constituir cadenas de valor territorial; así mismo es preciso volcar los esfuerzos hacia mecanismos mucho más sólidos de vinculación entre el diagnostico sectorial no descriptivo, sino estratégico, y el aporte del nivel cantonal en temas de uso y gestión del suelo y del nivel provincial, en líneas sobre el modelo productivo; y, por supuesto entre las intervenciones territoriales y sectoriales.

\section{Referencias bibliográficas.}

Ballesteros, N., Navia, A., y Romero, E., (2013). La política Medellín Ciudad Clúster, ¿contribuye al desarrollo endógeno? Una aproximación desde los cuatro planos de Boisier. DAAPGE 13 (20) 167 - 191.

Burgos, A.L. \& Bocco, G. (2020). Contribuciones a una teoría de la innovación rural. $\begin{array}{llll}\text { Cuadernos de } & \text { Economía, } & 39 & \text { (79), }\end{array}$ https://doi.org/10.15446/cuad.econ.v39n79.74459

Briceño, D. (2018). Construcción participativa de estrategias para el desarrollo endógeno de la vereda El Trigo, municipio de Guayabal de Síquima, Cundinamarca, Colombia, Inventum, 13(25), 2-11. https://doi.org/10.26620/uniminuto.inventum.13.25.2018.211

Canzanelli, G. (2004). Valorización del potencial endógeno, competitividad territorial y lucha contra la pobreza. CIRCLE. (1). https://bit.ly/34NdPZv

Cavuoto, N. (2009). Herramientas para planificar el desarrollo: un Sistema de Información Territorial con enfoque de cadena de valor. Territorios, 20-21, 175-205. https://bit.ly/2TVojSq

Código Orgánico de Planificación y Finanzas Públicas (COPYFP). (2010, 22 de octubre). Asamblea Nacional del Ecuador. Registro Oficial Suplemento No. 306. Segundo Suplemento. https://bit.ly/3nrozoh

Gobierno Autónomo Descentralizado (GAD) Malacatos (2018). Plan de Desarrollo y Ordenamiento Territorial, Parroquia Malacatos, Cantón Loja, Provincia de Loja. 
Gómez, G., Boada, A., y Alzate, I., (2018). Desarrollo endógeno y sustentable mediante un modelo de planificación estratégica. Caso de estudio: comuna Guaicamacuto, Venezuela. Espacios, 39 (32), 9. https://bit.ly/2XRPkZH

Instituto de Estadísticas y Censos [INEC]. (2010). VII Censo de Población y VI de Vivienda. https://bit.ly/3oo6Uiw

Massiris, A. (2015). "Hay que sostener la vida no la economía". Centro Internacional de Responsabilidad Social \& Sostenibilidad, http://www.mediafire.com/file/5zk6oh02grzaxh9/Revista+RS+Ed-1.pdf

Massiris, A. (2012). Gestión territorial y desarrollo. Hacia una política de desarrollo territorial sostenible en América latina. Universidad Pedagógica y Tecnológica de Colombia, Dirección de Investigaciones, Colección Investigación UPTC.

Massiris, A. (2020). Modelos de desarrollo territorial y ambiental. [presentación de diapositivas].

Moisá, L. y Hernández, A. (2010). El desarrollo rural de Medellín (Colombia) desde la perspectiva del desarrollo endógeno. Agronomía Colombiana, 28 (3), 515-523. https://revistas.unal.edu.co/index.php/agrocol/article/view/14417/28964

Quispe, G. (2016). Visiones del desarrollo endógeno desde las comunidades locales. PERSPECTIVAS, (37), 95-122. https://bit.ly/3nLy1D6

Quispe, G., y Ayaviri, V. (2013). Políticas de desarrollo en los procesos de desarrollo endógeno. Revista labor interdisciplinaria de desarrollo regional - Líder, (22) 151187. https://bit.ly/3cUyXPZ

Sáenz, A. (2006). Estándares para la medición de la gestión del desarrollo local. Cadernos EBAPE.BR, 4 (4), 1-30. https://bit.ly/3cSGfnn

Sánchez, L. (2015). Los problemas de la planificación y el desarrollo territorial en la comarca de Tabernas (Almería). Ager Revista de Estudios sobre Despoblación y Desarrollo Rural. (19). 148-180. https://www.redalyc.org/articulo.oa?id=29642953005

Secretaría Nacional de Planificación y Desarrollo (Senplades). 2015. Lineamientos para la Elaboración de Planes de Desarrollo y Ordenamiento Territorial Parroquiales.

Schejtman, A. (2010). Elementos para una renovación de las estrategias de desarrollo rural. Agronomía Colombiana, 28 (3), 445-454. https://bit.ly/2XhLHee

Torres, H. et. al. (2011). Alternativas De Vida: Trece Experiencias De Desarrollo Endógeno En Ecuador. [Tesis de maestría, Universidad Politécnica Salesiana]. Archivo digital https://bit.ly/2zvSAAs 
ISSN: 2600-5859

www.concienciadigital.org

Vol. 4, N¹.1, p. 101-123, Febrero, 2020

Valenciano, J. y Carretero, A. (2006). Desarrollo local en el mundo rural: El caso europeo. PERSPECTIVAS, 9

57-80.

https://www.redalyc.org/articulo.oa?id=425942413003

Vázquez, A. (2007). Desarrollo endógeno. Teorías y políticas de desarrollo territorial. Investigaciones Regionales - Revista de Investigación Regional, (11), 183-210. https://www.redalyc.org/articulo.oa?id=28901109

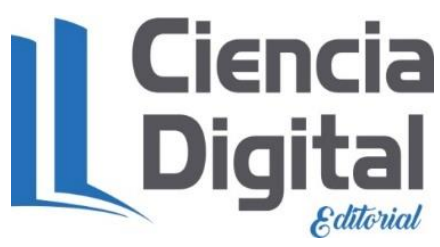




\section{PARA CITAR EL ARTÍCULO INDEXADO.}

Jiménez Aguilera, Z. N. (2021). Planificación territorial, una valoración desde las perspectivas del desarrollo rural endógeno en el caso de la parroquia Malacatos, del cantón Loja. ConcienciaDigital, $\quad 4(1.1), \quad 101-123$. https://doi.org/10.33262/concienciadigital.v4i1.1.1549

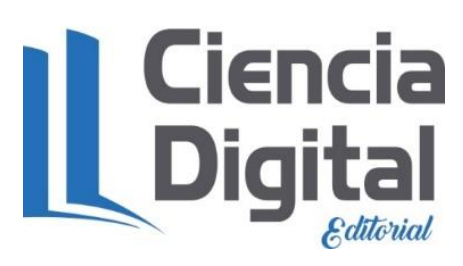

El artículo que se publica es de exclusiva responsabilidad de los autores y no necesariamente reflejan el pensamiento de la Revista Conciencia Digital.

El artículo queda en propiedad de la revista y, por tanto, su publicación parcial y/o total en otro medio tiene que ser autorizado por el director de la Revista Conciencia Digital.

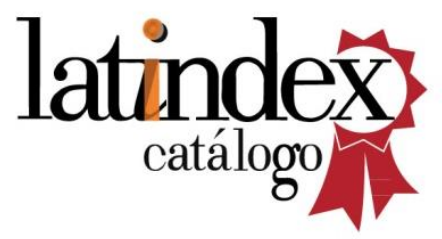

